\title{
SOFIA observatory performance and characterization
}

Temi, Pasquale NASA Ames Research Center United States pasquale.temi@nasa.gov

Miller, Walter Orbital Science Corp. United States Walter.E.Miller@nasa.gov

Dunham, Edward Lowell Observatory United States dunham@lowell.edu

McLean, Ian University of California, Los Angeles United States mclean@astro.ucla.edu

Wolf, Jurgen Deutsches SOFIA Institut Germany jwolf@sofia.usra.edu

Becklin, Eric USRA - SOFIA Science Center United States ebecklin@sofia.usra.edu

Bida, Tom Lowell Observatory United States tbida@lowell.edu

Brewster, Rick Orbital Science Corp. United States rick.brewster@nasa.gov

Casey, Sean USRA - SOFIA Science Center United States scasey@sofia.usra.edu

Collins, Peter Lowell Observatory United States plc@lowell.edu

Horner, Scott NASA Ames researc Center United States scott.horner@nasa.gov

Jakob, Holger DSI Deutsches SOFIA Institut Germany jakob@dsi.uni-stuttgart.de

Jensen, Stephen NASA Dryden Flight Research Center United States stephen.c.jensen@nasa.gov

Killebrew, Jana NASA - MSFC / Ames Research Center United States jana.I.killebrew@nasa.gov

Lampater, Ulrich DSI Deutsches SOFIA Institut Germany lampater@dsi.uni-stuttgart.de

Mandushev, Georgi Lowell Observatory United States gmand@lowell.edu

Marcum, Pamela NASA Ames Research Center United States pamela.m.marcum@nasa.gov

Meyer, Allan USRA - SOFIA Science Center United States ameyer@sofia.usra.edu

Pfueller, Enrico Deutsches SOFIA Institut United States epfueller@sofia.usra.edu

Reinacher, Andreas reinacher@dsi.uni-stuttgart.de

Roeser, Hans-Peter DSI Deutsches SOFIA Institut Germany roeser@irs.uni-stuttgart.de

Savage, Maureen USRA - SOFIA Science center United States msavage@sofia.usra.edu

Smith, Erin NASA Ames Research Center United States erin.c.smith@nasa.gov

Teufel, Stefan SOFIA Airborne System Operation Center United States teufel@dsi.uni-stuttgart.de

Wiedemann, Manuel SOFIA Deutsches SOFIA Institut United States mwiedemann@sofia.usra.edu

\section{ABSTRACT}

The Stratospheric Observatory for Infrared Astronomy (SOFIA) has recently concluded a set of engineering flights for Observatory performance evaluation. These in-flight opportunities have been viewed as a first comprehensive assessment of the Observatory's performance and will be used to address the development activity that is planned for 2012, as well as to identify additional Observatory upgrades. A series of 8 SOFIA Characterization And Integration (SCAI) flights have been conducted from June to December 2011. The HIPO science instrument in conjunction with the DSI Super Fast Diagnostic Camera (SFDC) have been used to evaluate pointing stability, including the image motion due to rigid-body and flexible-body telescope modes as well as possible aero-optical image motion. We report on recent improvements in pointing stability by using an Active Mass Damper system installed on Telescope Assembly. Measurements and characterization of the shear layer and cavity seeing, as well as image quality evaluation as a function of wavelength have been performed using the HIPO+FLITECAM Science Instrument configuration (FLIPO). A number of additional tests and measurements have targeted basic Observatory capabilities and requirements including, but not limited to, pointing accuracy, chopper evaluation and imager sensitivity. SCAI activities included in-flight partial Science Instrument commissioning prior to the use of the instruments as measuring engines. This paper reports on the data collected during the SCAI flights and presents current SOFIA Observatory performance and characterization. 